\title{
Thicker Funicular: Particle-Spring Systems for Variable-Depth Form-Responding Compression-Only Structures
}

\author{
B. Clifford \\ Massachusetts Institute of Technology SA+P, Boston, Massachusetts, United States of America
}

ABSTRACT: Particle-spring systems are commonly used to develop compression-only form finding systems. This paper proposes to use a particle-spring system to respond to a desired form in order to generate a variable-depth, compression-only structure. As a variable-depth system, loads can be re-directed through the depth of material in order to result in a desired form, as opposed to a structurally optimal form that assumes a uniform thickness approach. This paper proposes to generate, build, and test compression-only structures in response to a desired architectural geometry. This research will allow for integration with external programs to input a desired form, and result in a constructable compression-only structure.

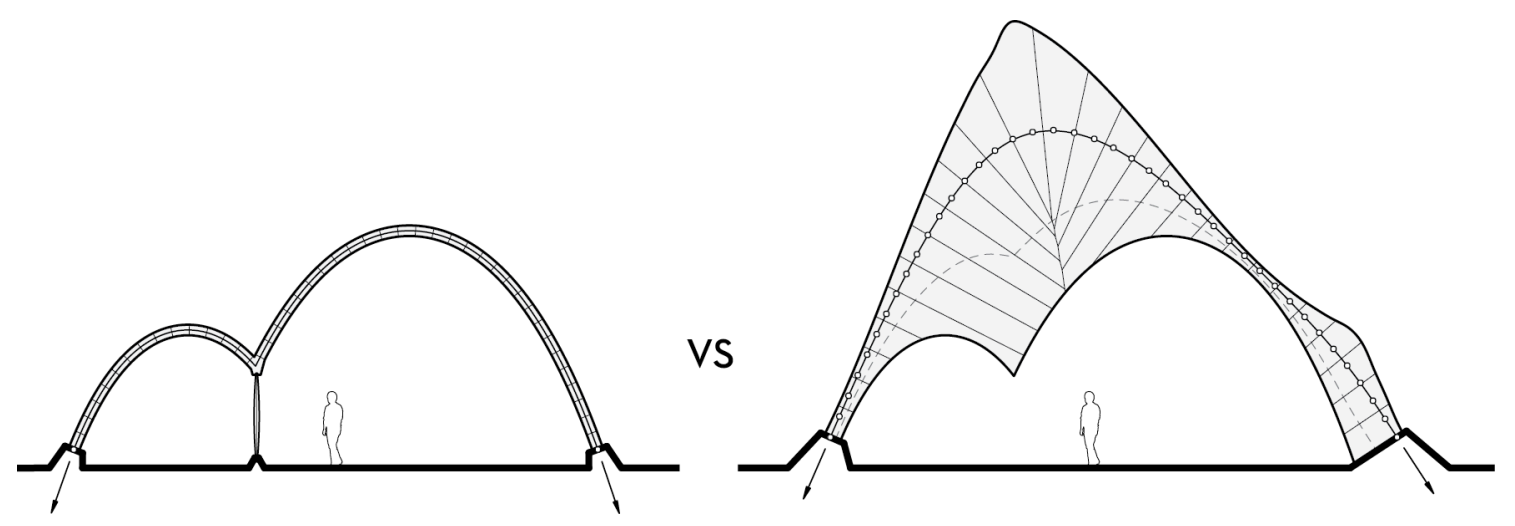

Figure 1. A demonstration of the difference between a thin funicular (left) and the proposed thick funicular (right). The thick funicular is able to accommodate the same bottom-geometry without the use of a compression rod.

Thin-shell compression-only structural systems are relatively new to the built environment. Compression-only structures on the other hand are ancient. Thin-shell structures assume a minimal and consistent cross-section. This assumption is driven by material efficiency. The results are forms developed exclusively by structural concerns (typically gravity), hence the term formfound. Architecture has to respond to structural concerns, but it also has to address a variety of other issues - acoustic, form, program, etc. It is not necessary for form to be driven strictly by structural requirements. For example, Gothic Cathedrals contain the thrust-vector within the variable depth of the stone's cross-section. These Cathedrals are not determined by idealized catenary form, but through a confluence of architectural desires with compression-only principles. With this approach as inspiration, this paper addresses the potentials of compression-only systems to be resolved through a variable-depth in order to obtain a desired form. 
Much research has been done in analyzing existing variable-depth structures to determine if a thrust vector falls inside the depth of material (Block et al, 2006). Other methods assume a fixed depth of material in order to generate a design. The method proposed in this paper assumes a desired geometry and allows for a variable thickness to re-direct the thrust vector as a means to produce a viable design that concerns both structure and other formal concerns. If typically one assumes thin, this paper assumes form.

This method is dedicated to addressing architectural concerns with structural results. This paper does not advocate for the reversion to a past architecture. It promotes the insertion of lost knowledge into our current means and methods of making.

\section{PARTICLE-SPRING SYSTEMS}

Particle-spring systems are based on lumped masses, called particles, which are connected to linear elastic springs. The solver used for this research is part of a particle-spring system implemented by Simon Greenwold (Fry and Reas, 2011). "Each particle in the system has a position, a velocity, and a variable mass, as well as a summarized vector for all of the forces acting on it" Kilian and Ochsendorf (2005). This Runge-Kutta solver is not necessary to generate a catenary (even load distribution), but it is necessary when evaluating an irregular load case. The method applied in this research will always be an irregular load case because it is assumed the resulting geometry is not an idealized catenary form.

Particle-spring systems have been explored to create virtual form-finding methods such as Kilian's CADenary tool (Kilian and Ochsendorf, 2005).

\section{COMPRESSION-ONLY STRUCTURES}

A compression-only structure will stand as long as the thrust vector of the system falls within the middle third of its cross section. It is possible for a system to stand if the thrust vector lands between the middle third and the outer surface; however, it is likely to develop a hinge. For the purposes of this paper, we will maintain a thrust vector inside the middle third of the structure, assuring a result with zero tension.

It is not always predictable that a structure will fail, though it is possible to know if it will stand. A paper (Heyman, 1966) introduced the safe theorem for masonry structures. This theorem states that a compression-only structure can stand so long as one network of compression forces can be found in equilibrium within the section of the structure. This solution is a possible lower-bound solution. When evaluating existing structures, it is not always possible to understand where exactly this force network is (Block and Ochsendorf, 2008). The method applied in this paper can calculate and assure a thrust vector falls within the thickness of material; however, it cannot guarantee one will not. Because of this uncertainty, a number of assumed failures did not fail.

For further reading on lower-bound analysis of unreinforced masonry structures, see (Heyman, 1982) and (Huera, 2001, 2004).

\section{FORM RESPONDING}

Form-finding analog models by such researchers as Otto and Gaudi, or even the virtual versions like Kilian's CADenary (Kilian and Ochsendorf, 2005) have proved it is difficult to control and predict the results of the final found-form. Moreover, if that form does not correspond with a force that is external to the form-finding model, it is difficult to resolve the two into a solution. This paper proposes form-responding as approach. Form-responding takes a desired form as input and produces a variable-depth to allow for interaction between these external forces and the solver-based model. 


\section{METHODOLOGY}

The method applied in this paper communicates a dynamic relaxation of the particle-spring system in response to a desired base-geometry. This system uses a solver-based approach to inform each particle in the system with the data it requires to compute a solution. The model can be described by the following categories-Base Geometry, Particle-Spring System, Vertical Distance, Expansion/Contraction, Unit Discritization.

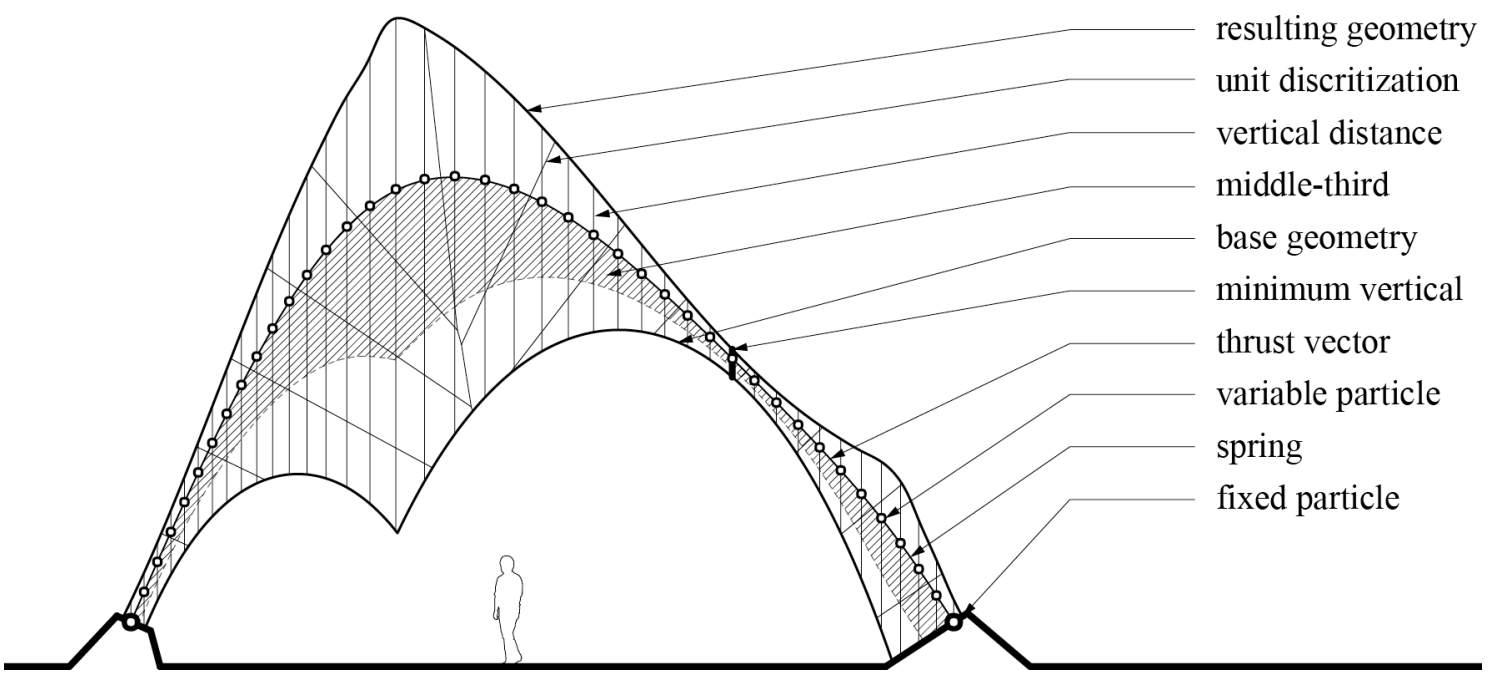

Figure 2. Key of the various components in the system

\subsection{Base Geometry}

This paper assumes the base geometry as fixed. The assumption is that this geometry has been pre-determined by a force external to the model-acoustics, formal, building-code, etc. Future research could allow for a more fluid and reciprocal relationship between the structural requirements and these other formal drivers. Not all geometries are currently possible. For instance, vertical undercut is not yet a possible geometry to solve with this method because the vertical intersection rule (see 4.3) does not calculate any mass below the first intersection. Another constraint is resolution. The more volatile this geometry is, the more likely it is for a particle to skip over a nuance in the geometry. Increasing the number of particles as a way to increase resolution can solve this. Smooth curves with a minimal change in radius tend to work best as they compensate for the discrepancies between two particles easily.

A base geometry is required as input to the system. This base geometry is the datum with which the particle system computes it-self against. Experiments have been conducted with both $2 \mathrm{D}$ curves and 3D surfaces.

\subsection{Particle-Spring System}

In Processing, a simple five-node b-spline serves as the base geometry. The middle node of this b-spline is interactive. By hovering with the curser, the clicking and dragging, it is possible for the user to manipulate the curve dynamically to interact with the system, and better understand how that geometry informs the particle-spring system. While this was a helpful demonstrative device, the dynamism is confusing. This paper proposes to create a hard geometry in which the particle system responds against. The interactivity is intended for demonstration, not as method.

The number of particles, the length of the springs, and the continual resulting forces on each particle inform the particle-spring system. While this organization is consistent, the system can be re-configured to produce a variety of potential solutions irrespective of the base-geometry. This model can produce 2D arches, (Clifford, 2011) but it can also produce aggregated arch vaults, networked mesh vaults, as well as composite solutions. 
The aggregated arch vaults are simply the aggregation of a series of independent arches in response to a single base-geometry. These arches are not dependant on the others to stand. This approach has been tested in both parallel and perpendicular configurations.

The networked mesh vault is a truly three-dimensional problem. While unwieldy and difficult to predict, it is possible to result in a solution with less material depth than the aggregated arch. The advantage the 2D system has is it is calculating a minimal cross section (see 4.4) across the particles of a single arch, where-as the mesh has to expand and contract the entire system until that minimum dimension is met.
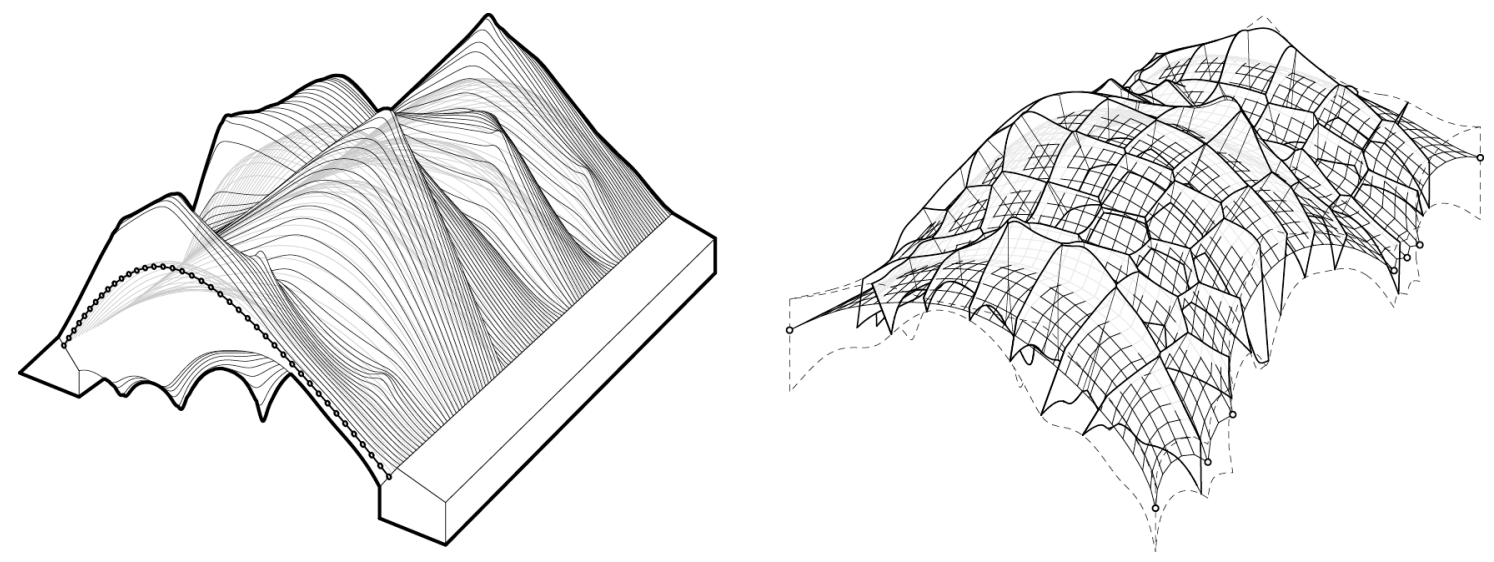

Figure 3. Drawings of an Aggregated Arch Vault (left) and a Networked Mesh Vault (right).

\subsection{Vertical Distance}

The spire of a Gothic Cathedral has a deeper cross section, and therefore contains more mass, re-directing the thrust vector down into its column. This model is similar in that the depth of material is highly variable producing a non-uniform load on the system (not a catenary). In order to simulate this, the model calculates a vertical intersection between particle and the basegeometry. Figure 4 demonstrates the distance between the particle and this intersection becomes the new vertical thrust vector for that particle. This calculation is continually calculated as the model finds equilibrium.

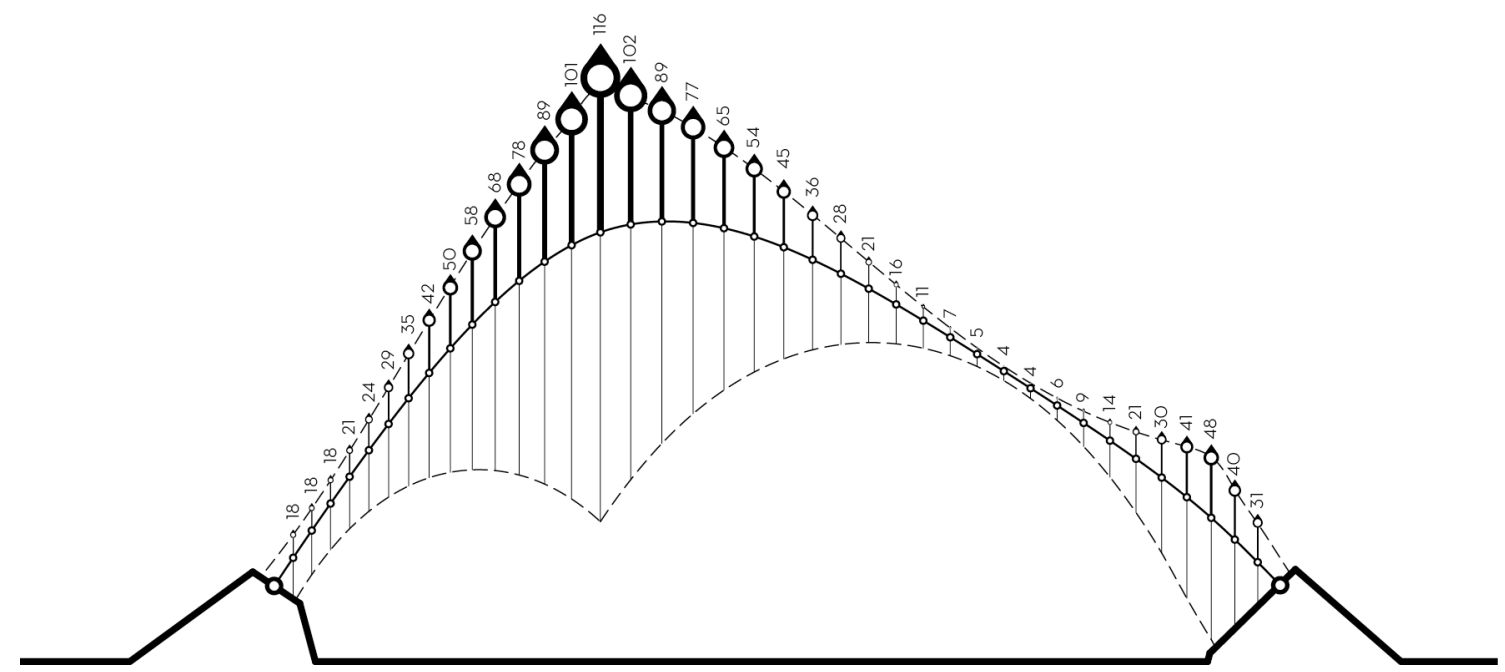

Figure 4. Diagram of the Vertical Distance calculation. The vertical distance between a particle and the base geometry equals the vertical thrust on that particle.

From the above $\mathrm{d}=\mathrm{F}$

where $\mathrm{d}=$ vertical distance between the particle and the base geometry; $\mathrm{F}=$ vertical force. 
When analyzing masonry arches, it is common practice to use static block analysis to break down an arch into a few polygons. The area of each polygon determines the vertical thrust vector (Block et al, 2006; Block and Ochsendorf, 2007). This paper relies on the high resolution of the particle-spring system informing each particle with its vertical distance.

\subsection{Expansion/Contraction}

In order to determine a solution where the thrust-vector falls above the base-geometry, the length of each spring needs to expand evenly throughout the system. It is possible to over expand the lengths, producing a viable solution with an extremely large mass, so a balance needs to be reached in order to find an efficient solution. The designer prescribes a minimal cross section dimension that can be informed by the compression capacity of the material. The system continually searches for the shortest vertical dimension. If that dimension is shorter or longer than the required minimum, the entire system expands or contracts. (Figure 2)

\subsection{Unit Discretization}

Once a desired solution is obtained, it is necessary to break the system down into constructible blocks. In order to resolve the equilibrium of shear forces (and not have to rely on friction) the break lines are created perpendicular to the thrust-vector. The number of breaks, and the regularity of these breaks do not alter the solution. When working with 3D systems, a thrust-network surface is created in order to generate break geometries normal to this surface. (Figure 3) As long as these breaks are perpendicular (normal) to the thrust vector, the system can assure a zero shear solution, meaning blocks can be placed without relying on glue or tension in the system.

\section{PROTOTYPING}

As this is a scale-less calculation, it has been possible to rapidly prototype each of the various configurations of the system at a small scale. These prototypes can be constructed from a variety of materials as long as they have a consistent density. The material must also be able to accept compression loads without deflection. Prototypes have been constructed from aggregated sheet materials and solid rapid prototyping for the three-dimensional calculations. (Figure 5,6)

Each prototype is erected without the aid of glue or fasteners. This verifies the system functions as a compression-only system. In order to demonstrate this, units are removed and the hinge is detected by recording the failure.
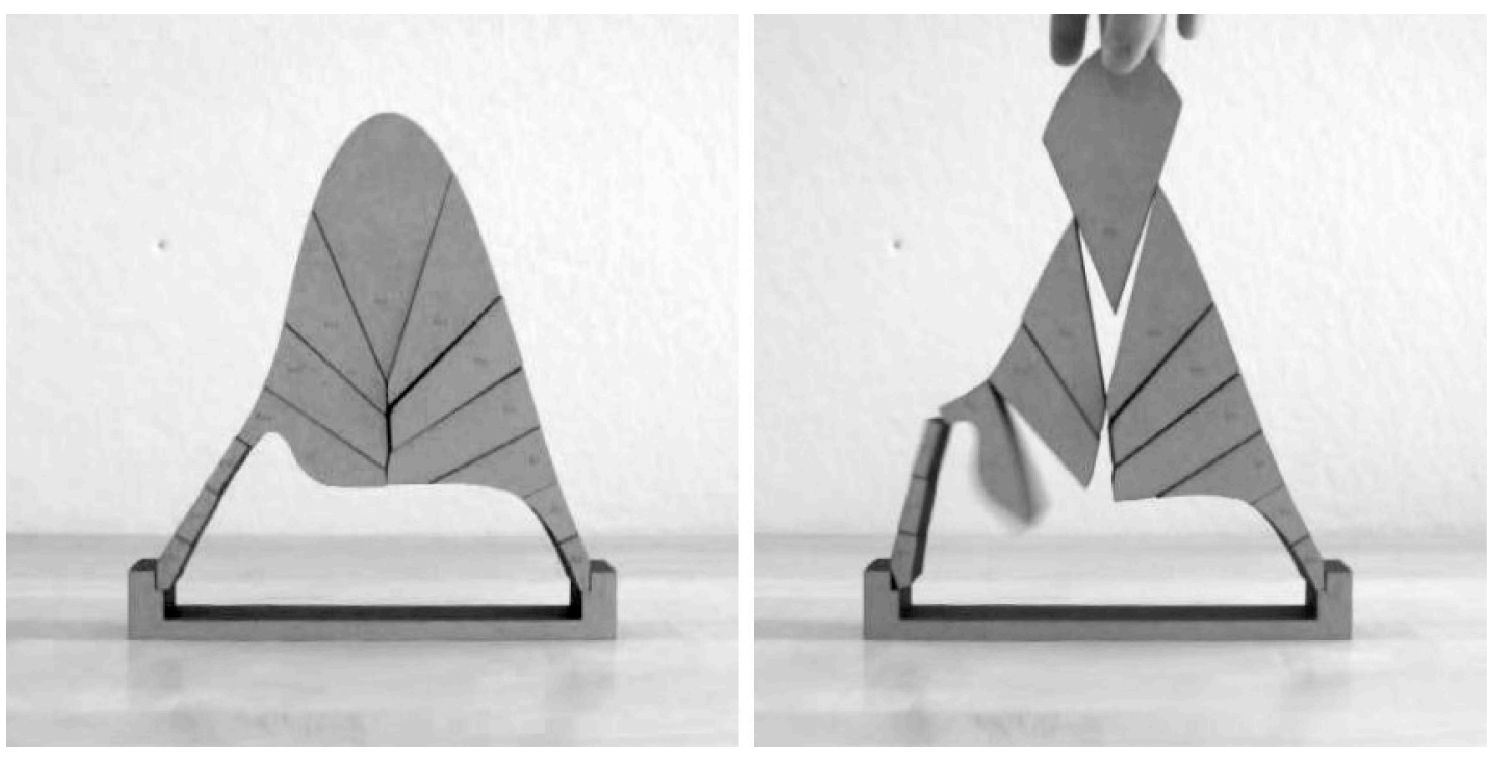

Figure 5. Prototype of a two-dimensional Arch. 


\subsection{Material}

It is possible to prototype these solutions from a variety of materials. Because the system is informing each particle with a linear vertical dimension, the only requirements are that the materials be of consistent mass in order to produce a relative result. It is also a requirement the material be able to accept compression loads without significant deflection. Prototypes were constructed from MDF sheet, Starch 3D prints, and plastic 3D prints.
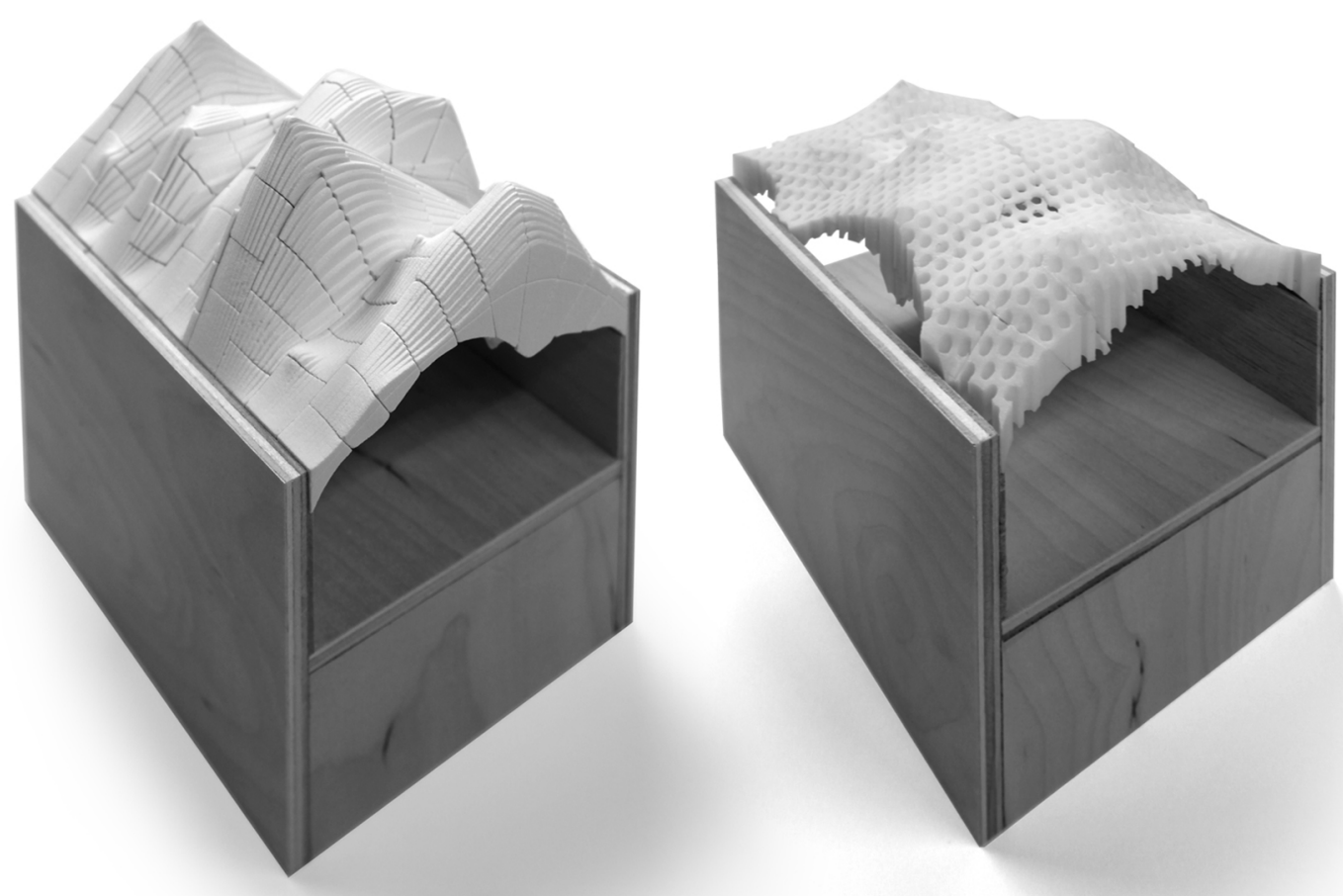

Figure 6. Prototypes of the Aggregated Arch Vault (left) and a Networked Mesh Vault (right).

\subsection{Erection Process}

Early prototypes were constructed with formwork dropped vertically after the units were placed. Later experiments negated the need for this formwork by incorporating a raceway for a string to post-tension and erect the system. (Figure 7) Once erect the string is removed to allow the thick funicular to maintain a compression-only solution.
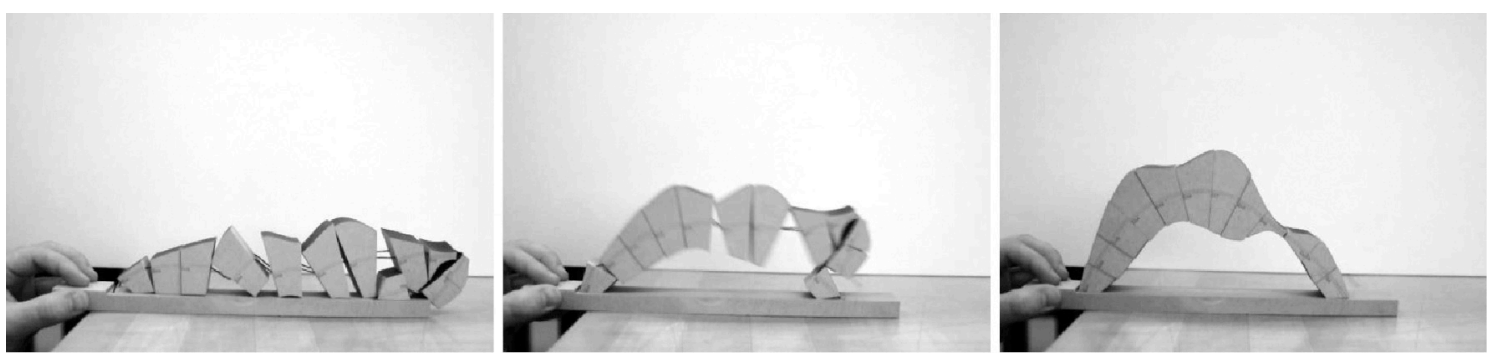

Figure 7. Post-tension erection prototype. This method negates the need for formwork. String is removed after erection. 


\section{ANALYSIS}

Not all of the prototypes would stand. Various theories were developed to explain why. These theories ranged from human error, to unit buckling in the z-direction. Videotaping these failures was helpful to play back in slow motion to verify the theories.

Additionally, some arches created specifically to demonstrate failure, did not fail. As mentioned previously, (see 2) as long as there is a lower bound solution, the arch will stand. This method currently guarantees a trust vector can fall within the thickness of material, but cannot guarantee one does not.

In order to verify the variable depth was contributing to ensuring the thrust vector remained inside the middle-third of the cross-section, it was necessary to produce a calculated failure. For this prototype, a pair of isolated loads informed a few particles on each side of the arch. These particles were overwritten in the code to understand they were to be two or sometimes four times more impacted by the vertical thrust. (Figure 8) When the mass was removed, the arch exposed a hinge point where the new thrust vector fell outside of the arch cross-section, demonstrating the variable mass was required to make the system stand.
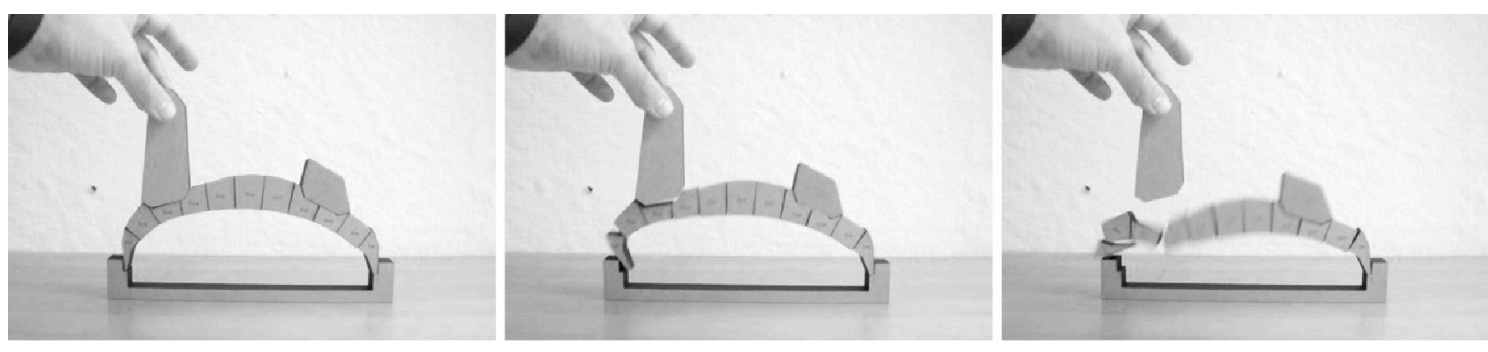

Figure 8. Prototype of an isolated external load case. By removing this load, the photo captures the hinges generated as the thrust vector leaves the thickness of the middle-third.

\section{CONCLUSION}

This paper demonstrates the potential to use a particle-spring system to generate a constructible compression-only structure that is not simply dedicated to material efficiently, but to the capacity to respond to other concerns architecture is required to address. While these concerns have not been inserted into this research, the placeholder (base-geometry) for these concerns is a venue for further exploration.

The system currently produces a single solution, though there are an infinite number of possible results. It is possible to expand and contract the system to accommodate varied minimum dimensions. It is also possible to add isolated load cases. Each variable is capable of producing a new solution. Future research could produce an array of solutions and select the one with the least mass or material as the most efficient solution.

The variable depth of the solutions also creates a number of problems with fabrication that need to be resolved. At the relatively small scale of these prototypes, aggregating sheet material and $3 \mathrm{~d}$ rapid prototyping is currently a placeholder for this problem of fabricating with volumetric materials. Future research will inform the model with the means and methods of making at full scale with materials such as AAC (aerated autoclaved concrete).

The results of this model are often extremely thick. While variable thickness is strongly advocated here, a question has arisen about the reality of this extreme depth change. Future research also resides in the potential to calculate a variable volume as opposed to thickness in order to accommodate a less exaggerated solution within a non-ideal funicular form. 


\section{ACKNOWLEDGEMENTS}

This paper presents results of an ongoing research project that began at the Princeton University School of Architecture under the tutelage of Axel Kilian and continued at The Ohio State University Knowlton School of Architecture (with Howard E. LeFevre Emerging Practitioner Fellowship funding) and the Massachusetts Institute of Technology (with Belluschi Lectureship funding). Two particle-spring systems have been used. The first was implemented by Simon Greenwold in Java as a library for "Processing" (www.processing.org), an environment developed by Ben Fry and Casey Rease. gHowl (www.grasshopper3d.com/group/ghowl) by Luis Fraguada was used to communicate via UPD between processing and Grasshopper (www.grasshopper3d.com), a plugin developed by David Rutten for Rhinoceros (www.rhino3d.com), a program developed by Robert McNeil. The second particle-spring system was generated entirely inside Grasshopper with the aid of two plugins - Kangaroo (www.grasshopper3d.com/group/kangaroo) by Daniel Piker served as the physics simulation of the particle-spring system and Hoopsnake (www.volatileprototypes.com/projects/hoopsnake) by Volitile Prototypes allowed the vertical distance to loop back into the calculation.

\section{REFERENCES}

Block, P., Ciblac, T. and Ochsendorf, J.: 2006, Real-time limit analysis of vaulted masonry buildings, Computers and Structures, 84(29-30), 1841-1852.

Block, P.: 2005, Equilibrium systems: studies in masonry structures, M.S. thesis, Cambridge, Massachusetts Institute of Technology.

Block, P.: 2009, Three-dimensional Equilibrium Approaches for Structural Analysis and Design, PhD Dissertation, Massachusetts Institute of Technology.

Block, P. and Ochsendorf, J.: 2008, Lower-bound analysis of unreinforced masonry vaults, in Fodde, E. (eds.), Proceedings of the VI International Conference on Structural Analysis of Historic Construction, CRC Press, 593-600.

Block, P. and Ochsendorf, J.: 2007, Thrust Network Analysis: A New Methodology for ThreeDimensional Equilibrium, Journal of the International Association for Shell and Spatial Structures, 48(3), 167-173.

Clifford, B.: 2011, Thick Funicular, Pidgin Magazine, 11, 220-239.

Clifford, B.: 2012, Thick Funicular, ACSA International Conference, forthcoming.

Heyman, J.: 1982. The Masonry Arch, Chickester: Ellis Horwood.

Heyman, J.: 1966, The Stone Skeleton, International Journal of Solids and Structures, 2, 249-279.

Huerta, S.: 2001, Mechanics of masonry vaults: the equilibrium approach, in Lourenço, P. and Roca, P. (eds.), Historical Constructions, Guimarães, 47-70.

Kilian, A. and Ochsendorf, J.: 2005, Particle-Spring Systems for Structural Form Finding, Journal of the International Association for Shell and Spatial Structures, 46(2), 77-84.

Maxwell, J.C.: 1864, On reciprocal figures and diagrams of forces, Phil. Mag.Series, 4(27), 250-261.

Moseley, H.: 1833, On a new principle in statics, called the principle of least pressure, Philosophical Magazine, 3, 285-288.

O’Dwyer, D.: 1999, Funicular analysis of masonry vaults, Computers \& Structures, 73(1-5), 187-197.

Otto, F. and Rasch, B.: 1995, Finding Form: Towards and Architecture of the Minimal, Berlin, Edition Axel Menges.

Rippmann, M. and Block, P.: 2011, Digital Stereotomy: Voussoir geometry for freeform masonry-like vaults informed by structural and fabrication constraints, Proceedings of the IABSE-IASS Symposium 2011, London, UK.

Rippmann, M. and Block, P.: 2011, New Design and Fabrication Methods for Freeform Stone Vaults Based on Ruled Surfaces, in Gengnagel, C., Kilian, A., Palz, N. and Scheurer, F. (ed.), Proceedings of Computational Design Modelling Symposium Berlin 2011, Springer, 181-189.

Block, P.: 2005, "Interactive Thrust". Available from: Open Source Repository $<$ http://web.mit.edu/masonry/interactiveThrust/> (accessed 01 November 2011).

Greenwold, S. and Allen, E.: 2003, "Active Statics". Available from: Open Source Repository $<$ http://acg.media.mit.edu/people/simong/statics/data/> (accessed 01 November 2011).

Cabrilog SAS: 2009, "Cabri Geometry II Plus". Available from: Open Source Repository $<$ http://www.cabri.com/> (accessed 01 November 2011).

Fry, B. and Reas, C.: 2011, "Processing web site". Available from: Open Source Repository $<$ http://www.processing.org/> (accessed 01 November 2011). 